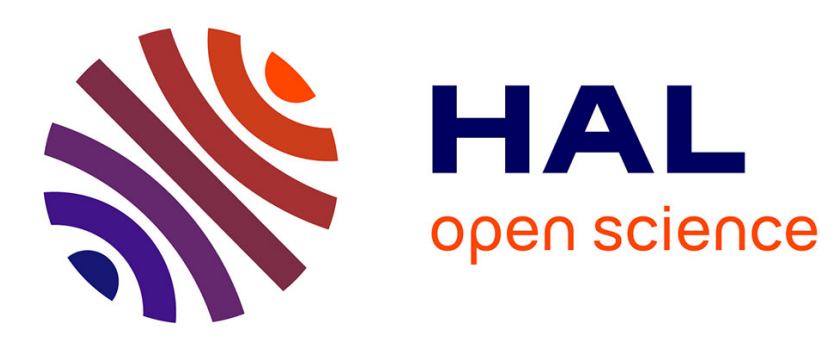

\title{
Role of vapor mass transfer in flow coating of colloidal dispersions in the evaporative regime
}

Charles Loussert, Frédéric Doumenc, Jean-Baptiste Salmon, Vadim Nikolayev, Beatrice Guerrier

\section{- To cite this version:}

Charles Loussert, Frédéric Doumenc, Jean-Baptiste Salmon, Vadim Nikolayev, Beatrice Guerrier. Role of vapor mass transfer in flow coating of colloidal dispersions in the evaporative regime. Langmuir, 2017, 10.1021/acs.langmuir.7b03297 . hal-01653411

\section{HAL Id: hal-01653411 https://hal.science/hal-01653411}

Submitted on 1 Dec 2017

HAL is a multi-disciplinary open access archive for the deposit and dissemination of scientific research documents, whether they are published or not. The documents may come from teaching and research institutions in France or abroad, or from public or private research centers.
L'archive ouverte pluridisciplinaire HAL, est destinée au dépôt et à la diffusion de documents scientifiques de niveau recherche, publiés ou non, émanant des établissements d'enseignement et de recherche français ou étrangers, des laboratoires publics ou privés. 


\title{
Role of vapor mass transfer in flow coating of colloidal dispersions in the evaporative regime
}

\author{
Charles Loussert, ${ }^{\dagger}$ Frédéric Doumenc, $^{*,+}, \uparrow$ Jean-Baptiste Salmon ${ }^{\dagger}{ }^{\top}$ Vadim S. \\ Nikolayev, ${ }^{\S}$ and Béatrice Guerrier ${ }^{\ddagger}$ \\ $\dagger$ CNRS, Solvay, LOF, UMR 5258, Univ. Bordeaux, F-33600 Pessac, France \\ $\ddagger$ Laboratoire FAST, Univ. Paris-Sud, CNRS, Université Paris-Saclay, F-91405, Orsay, \\ France \\ ФSorbonne Universités, UPMC Univ. Paris 06, UFR 919, 75005, Paris, France \\ $\S$ Service de Physique de l'État Condensé, CNRS, Université Paris-Saclay, CEA Saclay, \\ 91191 Gif-Sur-Yvette, France \\ E-mail: doumenc@fast.u-psud.fr \\ Phone: +331691537 31. Fax: +33169158060
}

\section{Abstract}

In flow-coating processes at low substrate velocity, solvent evaporation occurs during the film withdrawal and the coating process yields directly a dry deposit. In this regime, often referred to as the evaporative regime, several works performed on blade-coating-like configurations have reported a deposit thickness $h_{d}$ proportional to the inverse of the substrate velocity $V$. Such a scaling can be easily derived from simple mass conservation laws, assuming that evaporation occurs on a constant distance, referred to as the evaporation length, noted $L_{e v}$ in the present paper and of the order of the meniscus size. However the case of colloidal dispersions deserves further attention. Indeed, the coating flow leads to a wet film of densely-packed colloids before the formation of the dry deposit. This specific feature is related to the porous nature of the dry deposit, which can thus remain wet when capillary forces are strong enough to prevent the receding of the solvent through the pores of the film (the so-called pore-emptying). The length of this wet film may be possibly much larger than the meniscus size, therefore modifying the solvent evaporation rate, as well as the scaling $h_{d} \sim 1 / V$.
This result was suggested recently by different groups using basic modeling and assuming for simplicity an uniform evaporation rate over the wet film. In this article, we go a step further and investigate the effect of multi-dimensional vapor mass transfer in the gas phase on $L_{e v}$ and $h_{d}$ in the specific case of colloidal dispersions. Using simplified models, we first provide analytical expressions in asymptotic cases corresponding to $1 \mathrm{D}$ or $2 \mathrm{D}$ diffusive vapor transport. These theoretical investigations then led us to show that $L_{e v}$ is independent of the evaporation rate amplitude, and roughly independent of its spatial distribution. Conversely, $h_{d}$ strongly depends on the characteristics of vapor mass transfer in the gas phase, and different scaling laws are obtained for the 1D or the $2 \mathrm{D}$ case. These theoretical findings are finally tested by comparison with experimental results supporting our theoretical simplified approach.

\section{Introduction}

Flow coating techniques, such as knife-coating, blade-coating, or doctor-blade, have now become essential processes to coat continuously functional layers on solid substrates starting 
from dilute inks, and for applications ranging from organic electronics to optical coatings. ${ }^{1-3}$

Figure 1 shows schematically a typical bladecoating setup for the specific case of a colloidal dispersion. A liquid film is drawn out of a liquid reservoir confined between a fixed blade and a moving substrate at a velocity $V$. The fine
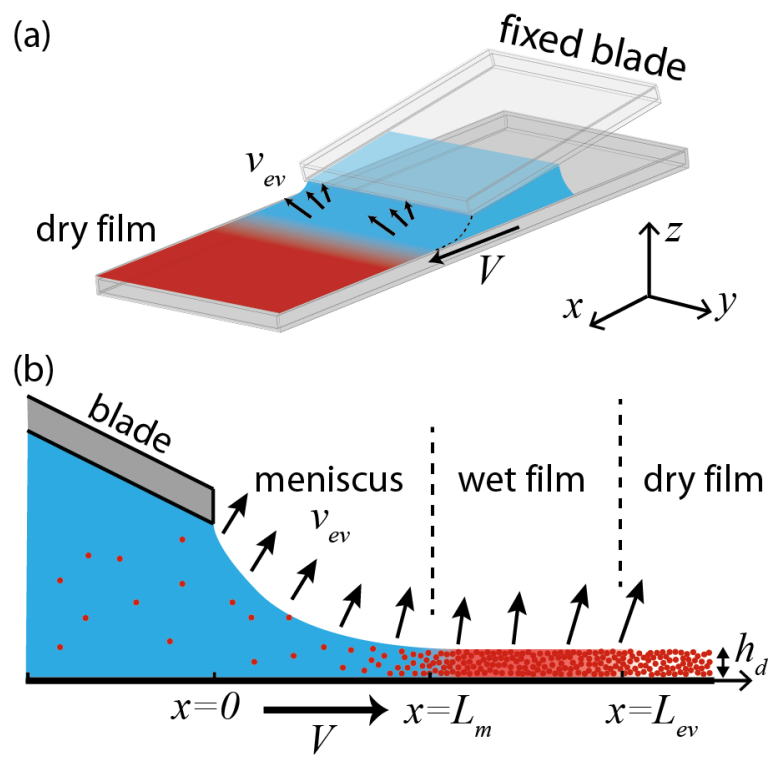

Figure 1: (a) Perspective view of a blade coating process in the evaporative regime. A dry film is continuously withdrawn from the reservoir by the substrate moving at a velocity $V$. (b) Side view evidencing the specific case of a colloidal dispersion. A solvent-saturated film is drawn out of a liquid reservoir and maintained by capillary forces between the blade and the moving substrate. Solidification occurs at a distance $x=L_{m}$, followed by a pore-emptying front at $x=L_{e v}$; arrows show schematically the local evaporative flux $v_{e v}(\mathrm{~m} / \mathrm{s}$, see text).

description of such flow-coating processes, and particularly the prediction of the final deposit thickness $h_{d}$ as a function of the process parameters and physico-chemical features of the ink, is still a major issue. Many works previously reported in the literature have clearly identified two regimes for such flow-coating-like processes depending of the coating speed $V \cdot{ }^{4-6}$ At high $V$, a liquid film is drawn out of the reservoir and dries later on. In this regime, often referred to as the Landau-Levich regime, drying and coating are separated in time, and the height of the liquid film results from a balance between surface tension and viscous friction induced by the substrate motion. ${ }^{7,8}$ At low $V$, solvent evaporation cannot be neglected during the film withdrawal, and the coating process yields directly a dry deposit: this is the evaporative regime.

In the last few years, many groups performed experimental and theoretical investigations of this evaporative regime for dilute inks ranging from colloidal dispersions ${ }^{5,9-14}$ to solutions of polymers and small molecules. ${ }^{4,14-16}$ Despite the wide complexity of the process and the wide variety of reported phenomena, including streaks or stripes formation, ${ }^{17-19}$ cracks and delamination $^{20}$ to name a few, many of the above cited works reported the following scaling:

$$
h_{d} \propto \frac{\varphi_{0}}{V},
$$

for the dry deposit thickness $h_{d}$ as a function of the substrate velocity $V$ and the reservoir particle volume fraction $\varphi_{0}$. Such a robust scaling suggests a common explanation based on mass conservation arguments only. Assuming that coatings are uniform along their width (invariance to translation along the $y$-direction, see Figure 1), simple mass balances for a binary mixture (volatile solvent + non-volatile solute) provide the following relation for the deposit thickness: ${ }^{9}$

$$
h_{d}=\frac{\varphi_{0}}{\varphi_{c}\left(1-\varphi_{0}\right)} \frac{Q_{e v}}{V}
$$

where $\varphi_{0}$ and $\varphi_{c}$ are the volume fractions of the non-volatile solute in the bulk and in the dry film, respectively, and $Q_{e v}\left(\mathrm{~m}^{2} / \mathrm{s}\right)$ is the solvent volume rate of evaporation per unit of width $W$. Eq (2) points out the important role played by the solvent mass transfer in the vapor phase to get a quantitative prediction of $Q_{e v}$ and thus $h_{d}$.

One recovers the scaling reported experimentally and given by eq (1) in the case of a dilute ink $\varphi_{0} \ll \varphi_{c}$ and assuming that $Q_{e v}$ does not depend on $\varphi_{0}, \varphi_{c}$ and $V$. This behavior was for instance observed in previous experiments performed in dip-coating-like configurations. ${ }^{4,5,14}$ $Q_{e v}$ was shown to depend only on drying air 
characteristics (temperature, humidity,...) and evaporation occurred over a constant length of the order of the meniscus size $L_{m}{ }^{14}$

Recently, several groups suggested that the case of colloidal dispersions is expected to be more intricate than the case of molecular solutes, and a significant departure from scaling (1) was even reported by Joshi and Gilchrist. ${ }^{21}$ Indeed, the coating flow first leads to a wet film of densely-packed colloids, which ultimately dries later on at a distance $L_{e v} \geq L_{m}$ from the static blade, see Figure 1(b). This specific feature is related to the porous nature of the dry deposit, which can thus remain wet when capillary forces are strong enough to prevent the receding of the solvent through the pores of the coating, the so-called poreemptying. ${ }^{22,23}$ In the case of colloidal dispersions, the evaporation length $L_{e v}$ now becomes a function of the process parameters, and can modify the solvent volume rate of evaporation $Q_{e v}$, as, in some cases, $L_{e v}$ can be much larger than the meniscus length $L_{m}$.

Jung and $\mathrm{Ahn}^{24}$ along with Joshi and Gilchrist $^{21}$ have considered Darcy's law to model solvent transport in the wet porous film and have assumed the uniformity of evaporation velocity $v_{e v}$ along the film, where $v_{e v}(\mathrm{~m} / \mathrm{s})$ is the local volume evaporation flux per unit of surface. This results in the expression

$$
Q_{e v} \simeq v_{e v} L_{e v}
$$

They further derived the following scaling

$$
h_{d} \propto \frac{\varphi_{0}^{2} v_{e v}}{V^{2}},
$$

for dilute dispersions $\varphi_{0} \ll \varphi_{c}$, assuming a criterion for estimating the pore-emptying front from the liquid pore pressure. The latter scaling fits correctly the data sets reported by Joshi and Gilchrist. ${ }^{21}$ However it fails to describe the experiments of refs ${ }^{5,11,14}$ which support scaling (1) for colloidal dispersions, as well as measurements of the evaporation length $L_{e v}$ reported by Jung and Ahn. ${ }^{24}$ However, in the theories ${ }^{21,24}$ evaporation is assumed to be dominated by the wet film only, i.e. $L_{e v} \gg L_{m}$, and the contribution of the meniscus to the overall evaporation rate $Q_{e v}$ is neglected. In a recent study, ${ }^{25}$ we included this contribution, still assuming uniform evaporation as above, and we recovered scalings (1) and (4) as two asymptotic limits of the same model, the latter corresponding to $L_{e v} \gg L_{m}$, the former to $L_{e v} \simeq L_{m}$. We further showed that this continuous model agrees with the measurements of $L_{e v}$ performed by Jung and Ahn, ${ }^{24}$ as their experiments were performed in the transition regime between scalings (1) and (4).

It is important to underline that all these models are based on the assumption of a uniform evaporation velocity $v_{e v}$ over the meniscus and the wet film. Indeed, eq (3) implicitly contains the assumption of $1 \mathrm{D}$ vapor mass transfer in the gas phase. Real configurations, such as the experimental case described in Figure 1, are expected to yield multi-dimensional mass transfer $(2 \mathrm{D}$ or even $3 \mathrm{D})$, with spatial variation of $v_{e v}$ all along the evaporation region. Indeed, the function $Q_{e v}\left(L_{e v}\right)$ should depend on the mechanisms which drive the vapor transport in the gas phase: free or forced convection, laminar or turbulent flow, etc. For instance, eq (3) would be recovered for turbulent free convection, from which proportionality between $L_{e v}$ and $Q_{e v}$ is expected, as derived by estimating mass transfer coefficients from empirical correlations (as available for instance in the book, ${ }^{26}$ and by using analogy between heat and mass transfer). In case of 3D vapor diffusion in quiescent air from a rectangular solvent puddle, a logarithmic dependence of $Q_{e v}$ on the evaporation length is expected (cf. section Theory for more details).

The main objective of the present article is to investigate the effect of multi-dimensional mass transfer in the gas phase on the evaporation length $L_{e v}$ and the deposit thickness $h_{d}$, for blade-coating of colloidal dispersions in the evaporative regime.

Using simplified models, we first provide analytical expressions of $L_{e v}$ and $h_{d}$ in asymptotic cases corresponding to $1 \mathrm{D}$ or $2 \mathrm{D}$ vapor transport. These theoretical investigations help us to show that $L_{e v}$ is independent of the evaporation intensity, and nearly independent of the mass transfer geometry, 1D or 2D. Conversely, 
the deposit thickness strongly depends on vapor mass transfer in the gas phase, and different scaling laws are obtained for the 1D and 2D cases.

We finally compare these theoretical predictions to experimental results obtained with a custom-made blade-coating setup for a wide range of parameters (concentration, colloid diameter, coating speed) and to data previously reported in the literature.

\section{Theory}

Building a model yielding an accurate description of a flow-coating-like process for colloidal dispersions in the evaporative regime is a hard task. Indeed, such a model should include a description of vapor mass transfer in the gas phase. Depending on the specific physical mechanism occurring in a given coating device, one should address a 3D diffusion problem if air is motionless, or a problem involving NavierStokes equations if the convection is relevant. Then this gas phase model must be coupled to governing equations of the liquid solvent flow within the meniscus and the wet film. Such an approach to our opinion is too complex and its feasibility currently questionable.

Regarding mass transfer in the gas phase, we use a simplified approach, based on analytical solutions of a 2D diffusion problem with a prescribed and constant boundary layer thickness $\Lambda$. We show in the next section that this 2D model qualitatively reproduces characteristic behaviour of more complex mass transfer mechanisms, from 3D diffusive transport to turbulent free convection. Therefore our approach, although simplified, is expected to provide physically relevant scaling laws for $L_{e v}$ and $h_{d}$.

\section{Mass transfer in the gas phase}

The 2D geometry on which our theoretical analysis is based is shown in Figure 1(b). Furthermore, we assume the existence of a boundary layer of constant thickness $\Lambda$ much larger than the height of the liquid film. We thus neglect the liquid height in the gas phase description and we use a simple rectangular geometry shown in Figure 2, with $0<x<\infty$ and $0<z<\Lambda$. To mimic the experimental geometry where a vertical wall blocks the vapor diffusion, we assume impermeability at the boundary $x=0$. The wet film extends from $x=0$ to $x=L_{e v}$, at $z=0$.

The 2D vapor concentration field $c(x, z)$ in the gas domain, shown in Figure 2, satisfies the Laplace equation $\partial_{x x} c+\partial_{z z} c=0$ with the boundary conditions:

$$
\begin{gathered}
x=0 \quad \text { and } \quad 0<z<\Lambda: \partial_{x} c=0 \\
z=0 \quad \text { and } \quad 0<x<L_{e v}: c=c_{s a t} \\
z=0 \quad \text { and } \quad x>L_{e v}: \partial_{z} c=0 \\
z=\Lambda \quad \text { and } \quad x>0: c=c_{\infty} .
\end{gathered}
$$

with $c_{\text {sat }}$ the saturated vapor concentration at the liquid/gas interface $\left(\mathrm{kg} / \mathrm{m}^{3}\right)$, and $c_{\infty}$ the ambient vapor concentration far from the interface $\left(\mathrm{kg} / \mathrm{m}^{3}\right)$.

The evaporation velocity in this wet film region $\left(0<x<L_{e v}\right)$ is given by the Fick's law $v_{e v}=-D_{v} / \rho \partial_{z} c$, where $D_{v}\left(\mathrm{~m}^{2} / \mathrm{s}\right)$ is the vapor diffusivity in air and $\rho$ the liquid solvent density $\left(\mathrm{kg} / \mathrm{m}^{3}\right)$. The volume rate of liquid which evaporates between the origin and any abscissa $x$ reads finally $Q_{e v}(x)=\int_{0}^{x} v_{e v}(\xi) d \xi$.

This problem is very similar to the one solved by Deegan et al for the coffee stain effect. ${ }^{27}$ These authors considered a 3D axi-symmetric geometry (liquid droplet), while we consider a 2D Cartesian geometry (liquid film). In the latter case, the problem in the upper half-space $(\Lambda \rightarrow \infty)$ has no solution in the steady state (see section 2.2 of $\operatorname{ref}^{28}$ for a demonstration). However a solution exists when the boundary condition (8) is prescribed at a finite height $\Lambda$.

The analytical solution of the Laplace equation with boundary conditions (5-8) has been obtained with a method of conformal transform. Calculations are detailed in Supporting Information (SI). The resulting evaporation ve- 


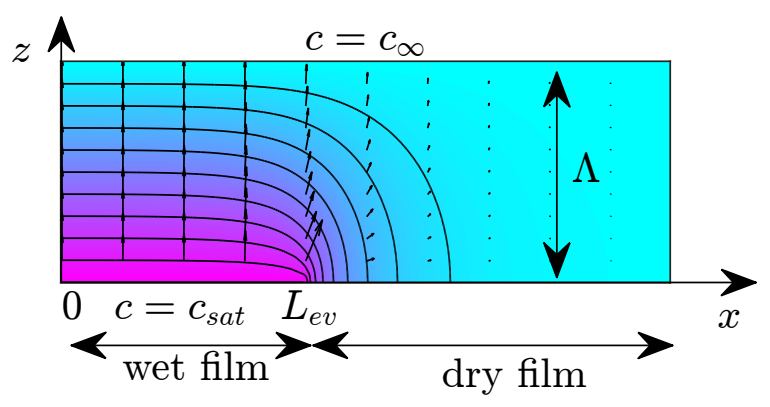

Figure 2: Gas domain. Solid lines represent isovalues of the concentration field $c(x, z)$ and arrows the diffusive flux.

locity $v_{e v}$ is

$$
\begin{gathered}
v_{e v}(x)=\frac{\pi \beta}{2 \Lambda K(k)}\left\{\left[\exp \left(\frac{\pi\left(L_{e v}+x\right)}{\Lambda}\right)-1\right]\right. \\
\left.\left[\exp \left(-\frac{\pi\left(L_{e v}+x\right)}{\Lambda}\right)-\exp \left(-\frac{2 \pi L_{e v}}{\Lambda}\right)\right]\right\}^{-1 / 2},
\end{gathered}
$$

where $\beta=D_{v}\left(c_{s a t}-c_{\infty}\right) / \rho, k=\exp \left(-\frac{\pi L_{e v}}{\Lambda}\right)$ and $K(k)$ is the complete elliptic integral of the first kind.

$v_{e v}$ and its integral $Q_{e v}\left(L_{e v}\right)$ are plotted in Figure 3. Depending on the value of the ratio $\Lambda / L_{e v}$, there are two asymptotic limits:

- $\Lambda / L_{e v} \ll 1$. The evaporation velocity is constant, except at the very end of the film, over a distance of the order of $\Lambda$. For the inner part of the film, such that $x \lesssim L_{e v}-\Lambda$, the diffusion problem reduces to the $1 \mathrm{D}$ case with a uniform evaporation velocity, and $v_{e v} \simeq \beta / \Lambda$, where

$$
\beta=D_{v} c_{\text {sat }}\left(1-H_{r}\right) / \rho,
$$

with $H_{r}=c_{\infty} / c_{s a t}$ the ambient relative humidity. The evaporation flux corresponding to this part of the film is thus proportional to the ratio $\left(L_{e v}-\Lambda\right) / \Lambda \simeq$ $L_{e v} / \Lambda$. It therefore diverges as $\Lambda / L_{e v} \rightarrow$ 0 .

Using eqs (S16-S17) of the SI, it can be shown that the evaporation flux corresponding to the very small zone at the tip of the film (of the order of $L_{e v}-\Lambda \lesssim$ $\left.x<L_{e v}\right)$ remains finite as $\Lambda / L_{e v} \rightarrow 0$ and can be neglected. The total evaporation flux is therefore

$$
Q_{e v}\left(L_{e v}\right) \simeq \beta \frac{L_{e v}}{\Lambda} .
$$

- $\Lambda / L_{e v} \gg 1$. The diffusion problem is fully $2 \mathrm{D}$. The total evaporation flux is now much less sensitive to $\Lambda / L_{e v}$, since it is inversely proportional to its logarithm:

$$
Q_{e v}\left(L_{e v}\right) \simeq \frac{\pi \beta}{2 \ln \left[16 \Lambda /\left(\pi L_{e v}\right)\right]}
$$

These two asymptotic regimes thus differ for two main reasons: (1) the local evaporation velocity $v_{e v}$ is nearly uniform in the $1 \mathrm{D}$ case, but varies all along the evaporation length in the $2 \mathrm{D}$ case; (2) the total evaporation flux is proportional to the evaporation length $L_{e v}$ in the 1D case, while it depends weakly on $L_{e v}$ in the $2 \mathrm{D}$ case.

It is worth noting that our simplified model qualitatively reproduces limit cases that would be expected with more detailed descriptions of vapor mass transfer. Indeed, eqs (11) and (3) both exhibit proportionality between $L_{e v}$ and $Q_{e v}$. This strong dependence of $Q_{e v}$ on $L_{e v}$ may represent turbulent free convection as stated in the introduction.

A weak logarithmic dependence similar to eq (12) may be obtained in 3D diffusion configuration. Indeed 3D vapor diffusion in quiescent air from a rectangular solvent puddle of size $L_{e v} \times W$, a geometry close to the case depicted in Figure 1, would give a volume evaporation rate $^{26}$

$$
Q_{e v}=\frac{\pi \beta}{\ln \left(4 W / L_{e v}\right)},
$$

with the assumption $L_{e v} \ll W$ and using equivalence between heat and mass diffusion.

In the following, we investigate the consequences of the two above asymptotic cases (1D and 2D) on the scaling laws for the evaporation length $L_{e v}$ and the deposit thickness $h_{d}$. 


\section{Governing equations in the liquid phase}

The global and particle mass balances provide a relation between the deposit thickness $h_{d}$ and the evaporation flux $Q_{e v}\left(L_{e v}\right):{ }^{4,9,25}$

$$
h_{d}=\frac{\varphi_{0}}{\varphi_{c}\left(1-\varphi_{0}\right)} \frac{Q_{e v}\left(L_{e v}\right)}{V} .
$$
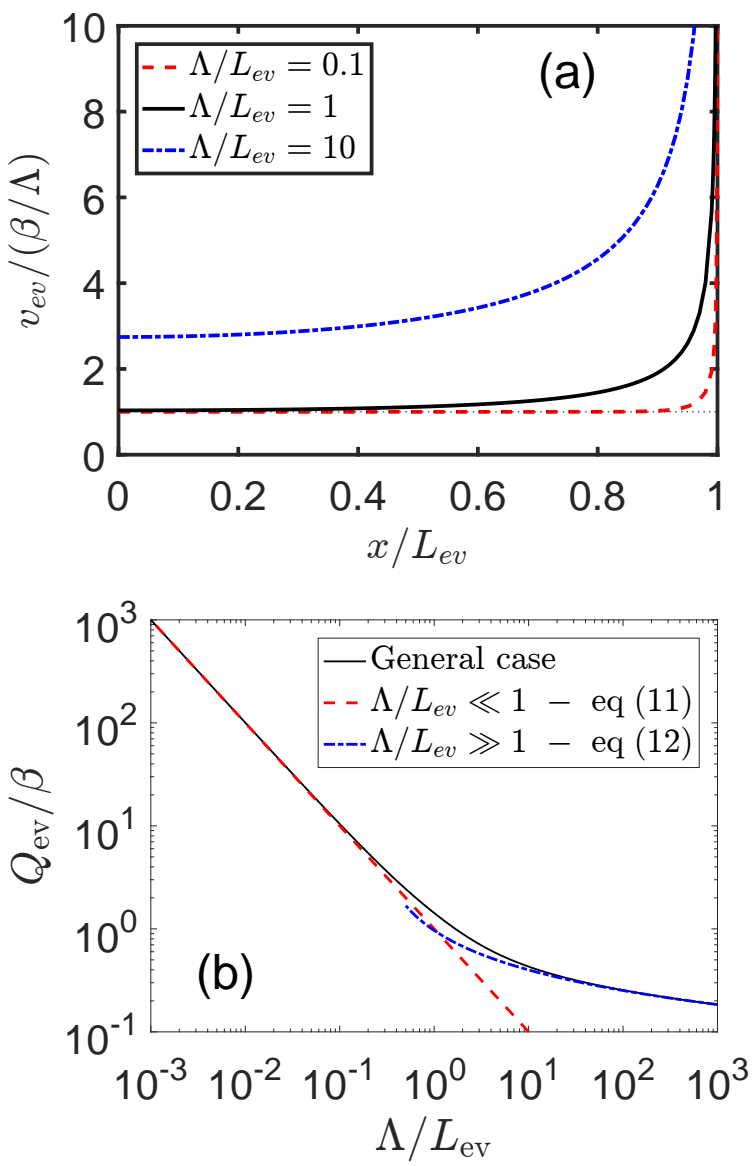

Figure 3: (a) local evaporation velocity $v_{e v}$ as a function of $x / L_{e v}$ for different values of $\Lambda / L_{e v}$ [from eq (9)]. (b) total evaporation volume rate $Q_{e v}$ as a function of $\Lambda / L_{e v}$; the general case (black solid line) corresponds to numerical integration of eq (9).
Eq (14) contains an implicit relation between two unknowns, the deposit thickness $h_{d}$ and the evaporation length $L_{e v}{ }^{1}$. A second relation can be derived from the Darcy's law and the solvent mass conservation in the wet film region (cf. Figure 1):

$$
\begin{gathered}
\partial_{x} p_{l i q}=-\frac{\eta_{0}}{k\left(\varphi_{c}\right)}\left(1-\varphi_{c}\right)(u-V), \\
h_{d}\left(1-\varphi_{c}\right) \partial_{x} u=-v_{e v}(x),
\end{gathered}
$$

where $p_{\text {liq }}, \eta_{0}$ and $u$ are the solvent pressure, viscosity and velocity, respectively, $\varphi_{c}$ is the closepacking particle volume fraction. We used the Koseny-Carman equation ${ }^{29}$ for estimating the permeability of the densely-packed wet coating

$$
k\left(\varphi_{c}\right)=\frac{2 a^{2}\left(1-\varphi_{c}\right)^{3}}{90 \varphi_{c}^{2}},
$$

where $a$ is the particle radius. Eq (17) is empirical for random packings; it also predicts the permeability of periodic arrays of spheres with an error less than $30 \%$ at high volume fraction. ${ }^{30}$

Since $L_{e v}$ is unknown, the resolution of eqs (15-16) requires three boundary conditions. A first condition is obtained at $x=L_{m}$ assuming that the capillary pressure in the wet film, fixed by the pore size of the order of the particle radius $a$, is much larger than the capillary pressure in the meniscus of macroscopic curvature $C_{0}$. We thus make the approximation

$$
p_{l i q}\left(L_{m}\right)-p_{a t m}=0
$$

\footnotetext{
${ }^{1}$ Note that we derived previously in our earlier work a slightly different relation $\left[h_{d} V\left(\varphi_{c}-\varphi_{0}\right)=\right.$ $\left.\varphi_{0} Q_{e v}\right]$ as we developed deliberately a continuous model solvent+colloids. Both relations however are equivalent for dilute dispersions $\varphi_{0} \ll \varphi_{c}$.
} 
where the meniscus length $L_{m}$ is assumed to be constant, and equal to its value when there is no flow (this last assumption has been validated by numerical simulations, see $\operatorname{ref}^{25}$ ). Two other conditions are obtained at $x=L_{e v}$. Assuming a negligible flux of water into the dry film yields

$$
u\left(L_{e v}\right)-V=0,
$$

and pore-emptying occurs for a critical capillary pressure given by

$$
p_{l i q}\left(L_{e v}\right)-p_{a t m}=-\kappa \gamma / a \text {. }
$$

For monodisperse spheres, we use

$$
\kappa=3 \varphi_{c} /\left(1-\varphi_{c}\right)
$$

as suggested in ref. ${ }^{31}$

Solving eqs (15-16) with boundary conditions (18-20) yields

$$
\begin{aligned}
h_{d}=\frac{a \eta_{0}}{\kappa \gamma k}\left[\left(L_{e v}-L_{m}\right) Q_{e v}\left(L_{e v}\right)\right. \\
\\
\left.\quad-\int_{L_{m}}^{L_{e v}} Q_{e v}(x) d x\right] .
\end{aligned}
$$

We introduce now the dimensionless quantities $\tilde{x}=x / L, \tilde{L}_{m}=L_{m} / L, \tilde{L}_{e v}=L_{e v} / L$, $\tilde{\Lambda}=\Lambda / L, \tilde{h}_{d}=h_{d} / h$ and $\tilde{Q}_{e v}=Q_{e v} / Q$. The scales $L, h$ and $Q$ are defined by taking as a reference the case corresponding to $L_{e v}=L_{m}$. We thus get $L=L_{m}, h=\varphi_{0} Q /\left[\varphi_{c}\left(1-\varphi_{0}\right) V\right]$, and $Q=\int_{0}^{L_{m}} v_{e v}(x) d x$ where $L_{m}$ has been substituted to $L_{e v}$ in the expression of $v_{e v}(x)$ in eq $(9)$.

Using eqs (14) and (22) to eliminate $h_{d}$ and turning to dimensionless variables, we get the equation to be solved to get $\tilde{L}_{e v}$ :

$$
\tilde{L}_{e v}-\frac{1}{\tilde{Q}_{e v}\left(\tilde{L}_{e v}\right)} \int_{1}^{\tilde{L}_{e v}} \tilde{Q}_{e v}(\tilde{x}) d \tilde{x}=1+\mathcal{M}
$$

where

$$
\begin{aligned}
\mathcal{M}=\frac{\varphi_{0}}{\varphi_{c}\left(1-\varphi_{0}\right)} & \frac{\kappa \gamma k}{V \eta_{0} a L_{m}} \\
= & \frac{\varphi_{0}}{1-\varphi_{0}} \frac{\gamma / \eta_{0}}{V} \frac{2 a}{L_{m}} f\left(\varphi_{c}\right)
\end{aligned}
$$

and $f\left(\varphi_{c}\right)=\left(1-\varphi_{c}\right)^{2} /\left(30 \varphi_{c}^{2}\right)$. The dimensionless number $\mathcal{M}$ compares the pore pressure at pore-opening $(\kappa \gamma / a)$ to the scale of the evaporation-induced pressure drop in a wet film of length $L_{m} \cdot{ }^{25} \mathcal{M}$ depends on the process geometry, dispersion properties and substrate velocity but not on the vapor mass transfer in the gas phase. Thus it may be easily estimated for a given configuration. As seen in the experimental results, it varies on several orders of magnitude in our experiments.

We see from eq (23) that $\tilde{L}_{e v}$ does not depend on evaporation intensity, but only on the shape of the function $\tilde{Q}_{e v}(x)$ [indeed, multiplying $\tilde{Q}_{e v}(x)$ by a constant has no effect on eq (23)]. The reason is that increasing the overall evaporation rate increases in the same proportion the deposit thickness and the liquid flow rate through the wet film. These two effects compensate each other, and the pressure drop in the wet film is unchanged [see Eqs (15)-(16)].

Eq (23) can be solved numerically to get $\tilde{L}_{e v}$. Once $\tilde{L}_{e v}$ is known, the deposit thickness $\tilde{h}_{d}$ is obtained from the dimensionless version of eq (14):

$$
\tilde{h}_{d}=\tilde{Q}_{e v}\left(\tilde{L}_{e v}\right)
$$

\section{Asymptotic regimes}

We first examine simplifications of eq (23) for the $1 \mathrm{D}$ and $2 \mathrm{D}$ cases.

- $\tilde{\Lambda} / \tilde{L}_{e v} \ll 1$ (1D case). From eq (9), $Q_{e v}(x) \simeq \beta x / \Lambda$. Eq $(23)$ reduces to a second degree algebraic equation whose solution is

$$
\tilde{L}_{e v} \simeq(1+\mathcal{M})+\sqrt{(1+\mathcal{M})^{2}-1}
$$

This is the solution already found in ref. ${ }^{25}$

- $\tilde{\Lambda} / \tilde{L}_{e v} \gg 1$ (2D case). The integration of eq (9) yields

$$
Q_{e v}(x) \simeq \beta \frac{\arcsin \left(x / L_{e v}\right)}{\ln \left[16 \Lambda /\left(\pi L_{e v}\right)\right]} .
$$

Plugging eq (27) into eq (23) provides an 
equation for $\tilde{L}_{e v}$ :

$$
\sqrt{\tilde{L}_{e v}^{2}-1}+\arcsin \left(1 / \tilde{L}_{e v}\right) \simeq \frac{\pi}{2}(1+\mathcal{M}) .
$$

Table 1 summarizes the analytical expressions of $\tilde{L}_{e v}$ corresponding to the four asymptotic cases obtained by combining eq (26) and eq (28) with additional assumptions $\mathcal{M} \ll 1$ or $\mathcal{M} \gg 1$. There is no difference between $1 \mathrm{D}$ and $2 \mathrm{D}$ cases for $\mathcal{M} \ll 1$. For $\mathcal{M} \gg 1, \tilde{L}_{e v}$ is proportional to $\mathcal{M}$ in both cases, and numerical prefactors differ only by $20 \%$ (for a confirmation, cf. solid and dashed lines in Figure 5(b), in the section dedicated to the comparison with experimental results).

These results lead to the following conclusions about the evaporation length. We already pointed out that evaporation intensity has no effect on $\tilde{L}_{e v}$; we see now that $\tilde{L}_{e v}$ is also weakly sensitive to the distribution of evaporation velocity along the wet film. In practice, the simple eq (26), although obtained in 1D, provides a reasonable estimate of $\tilde{L}_{e v}$ when applied to a 2D problem.

Table 1: Analytical expressions of the dimensionless evaporation length $\tilde{L}_{e v}=L_{e v} / L_{m}$ for all asymptotic cases.

\begin{tabular}{c|c|c} 
& $\Lambda / L_{e v} \ll 1$ & $\Lambda / L_{e v} \gg 1$ \\
& $(1 \mathrm{D}$ case $)$ & $(2 \mathrm{D}$ case $)$ \\
\hline $\mathcal{M} \ll 1$ & $\tilde{L}_{e v}=1$ & $\tilde{L}_{e v}=1$ \\
\hline $\mathcal{M} \gg 1$ & $\tilde{L}_{e v}=2 \mathcal{M}$ & $\tilde{L}_{e v}=\frac{\pi}{2} \mathcal{M} \simeq 1.6 \mathcal{M}$ \\
\hline
\end{tabular}

Analytical expressions of $h_{d}$ deduced from eq (25) are reported in Table 2 for all asymptotic cases. Scaling (4) (deposit thickness varying as $\left.1 / V^{2}\right)$ is recovered for the $1 \mathrm{D}$ case $\left(\Lambda / L_{e v} \ll 1\right)$ at $\mathcal{M} \gg 1$. It corresponds to the proportionality between $L_{e v}$ and $Q_{e v}$. In all other situations, a scaling more or less consistent with eq (1) (deposit thickness varying as $1 / V)$ is recovered. Eq (1) is obtained for $\mathcal{M} \ll 1$. For the $2 \mathrm{D}$ case $\left(\Lambda / L_{e v} \gg 1\right)$ at $\mathcal{M} \gg 1, h_{d}$ weakly depends on the terms under the logarithm, and their effect can hardly be detected experimentally. So for this case too, scaling (1) is expected in experiments. Particle size affects the deposit thickness in the case $\mathcal{M} \gg 1$ only. The dependence is significant in the $1 \mathrm{D}$ case (proportionality) but small in the 2D case (logarithmic term).

As a conclusion on the role of vapor mass transfer in the evaporative regime, these asymptotic models show that, unlike $L_{e v}$, the behavior of $h_{d}$ depends on the regime occurring in the gas phase.

\section{Experimental results}

\section{Experiments}

To check the validity of the above theoretical scalings, we developed a blade-coating setup. We measured both $L_{e v}$ and $h_{d}$ for a wide range of coating speeds $(V)$ and for different model colloidal dispersions, more precisely monodisperse charge-stabilized latex nanoparticles of different sizes and volume fractions (ThermoFisher and Sigma Aldrich Latex beads (PS), dilution with deionised MilliQ ${ }^{\mathrm{TM}}$ water), see Table 3. All our experiments were performed at room temperature $T=19.5 \pm 1^{\circ} \mathrm{C}$, and for external relative humidities $H_{r}$ ranging from $35 \%$ to $60 \%$. Eq (10) gives $\beta \simeq$ $(2.3 \pm 0.7) \times 10^{-10} \mathrm{~m}^{2} . \mathrm{s}^{-1}$, using $D_{v}=2.5 \times$ $10^{-5} \mathrm{~m}^{2} \cdot \mathrm{s}^{-1}$ and $\rho=997 \mathrm{~kg} \cdot \mathrm{m}^{-3} . c_{\text {sat }}$ is derived from the ideal gas law with the water saturated vapor pressure $P_{v s}=2300 \mathrm{~Pa}$ at $19.5^{\circ} \mathrm{C}$.

Figure 4(a) shows schematically our experimental setup. It consists in a microscope slab (thickness $\simeq 170 \mu \mathrm{m}$ ) glued on a thicker glass slab (thickness $\simeq 1 \mathrm{~mm}$ ) for mechanical stability and fixed to a first translation stage along z-axis (labeled 1 on Figure 4) to control the reservoir height $H$. Indeed, this stage is used to confine a small amount of colloidal dispersions $(1 \mathrm{~m} \ell)$ between the blade and a glass substrate holded on another translation motorized stage moving along x-axis at speed $V$ (labeled 2 on Figure 4, Märzhäuser Wetzlar). This motorized stage is itself mounted on another motorized translation stage along $\mathrm{x}$-axis supporting the blade setup (labeled 3 on Figure 4) which 
Table 2: Analytical expression of deposit thickness $h_{d}$ for all asymptotic cases.

\begin{tabular}{c|c|c} 
& $\Lambda / L_{e v} \ll 1(1 \mathrm{D}$ case $)$ & $\Lambda / L_{e v} \gg 1(2 \mathrm{D}$ case $)$ \\
\hline $\mathcal{M} \ll 1$ & $h_{d}=\frac{\varphi_{0}}{\varphi_{c}\left(1-\varphi_{0}\right)} \frac{\beta}{V} \frac{L_{m}}{\Lambda}$ & $h_{d}=\frac{\varphi_{0}}{\varphi_{c}\left(1-\varphi_{0}\right)} \frac{\beta}{V} \frac{\pi}{2 \ln \left[\frac{16 \Lambda}{\pi L_{m}}\right]}$ \\
\hline $\mathcal{M} \gg 1$ & $h_{d}=2 a \frac{\varphi_{0}^{2}}{\varphi_{c}\left(1-\varphi_{0}\right)^{2}} \frac{\beta}{V^{2}} \frac{\gamma f\left(\varphi_{c}\right)}{\eta_{0} \Lambda}$ & $h_{d}=\frac{\varphi_{0}}{\varphi_{c}\left(1-\varphi_{0}\right)} \frac{\beta}{V} \frac{\pi}{2 \ln \left[\frac{16 \Lambda}{\pi^{2}}\left(2 a \frac{\varphi 0}{1-\varphi_{0}} \frac{\gamma / \eta_{0}}{V} f\left(\varphi_{c}\right)\right)^{-1}\right]}$ \\
\hline
\end{tabular}

is fixed on an inverted microscope (Olympus, IX71). This original configuration allows us to monitor the coating process in-situ with the accuracy of bright field microscopy, and all along the coating by moving stage 3 over its translation range $(50 \mathrm{~mm})$.

For each experiment, we proceed as follows. We first clean the glass substrate (width = $50 \mathrm{~mm}$, length $=75 \mathrm{~mm}$ and thickness $=1 \mathrm{~mm}$ ) using a Piranha treatment (20 minutes in a $50 / 50$ vol. sulfuric acid/hydrogen peroxide solution, storage in MilliQ water after rinse) to ensure reproducible hydrophilic properties. We then confine a small volume of a colloidal dispersion $(1 \mathrm{~m} \ell)$ as shown schematically in Figure 4 , and a static meniscus of typical size $L_{m} \approx 100 \mu \mathrm{m}$ is formed for $H=500 \mu \mathrm{m}$ all along the width $W$ of the substrate. The typical angle that forms the blade with the glass substrate is of a few degrees, the thickness of the coating blade is $170 \mu \mathrm{m}$, and its width is $40 \mathrm{~mm}$. We then apply a constant speed $V=500 \mu \mathrm{m} / \mathrm{s}$ to deposit a first coating on a distance $\approx 5 \mathrm{~mm}$ in order to avoid transient and edge effects (pining of the line for instance), and we then apply a constant $V$ to coat the remaining blade, typically on a length $\approx 35 \mathrm{~mm}$. We then monitor the steady coating process using a 1.25X objective (Olympus) on a sCMOS camera (OrcaFlash, Hamamatsu) with a field of view of $1.3 \times 1.8 \mathrm{~mm}^{2}$. We finally use stage 3 (synchronised with the image acquisition) to acquire composite images over a wider field of view, typically $5.2 \times 1.8 \mathrm{~mm}^{2}$.

Figure 4(b) shows a typical example of such an extended image for set \# 3 listed in Table 3 $(V=5 \mu \mathrm{m} / \mathrm{s})$. The transition from the wet film to the the dry coating occurs at a distance
$L_{e v}=2100 \pm 300 \mu \mathrm{m}$ in this case, as easily observed using the change of refractive index owing to the invasion of the pores by air. In some conditions, the coatings display regular stripes (with typical wavelength $300 \pm 20 \mu \mathrm{m}$ ) which actually correspond to modulations of the deposit thickness of the order of a few $\mu \mathrm{m}$, see later. After full coating, the mean final thickness $h_{d}$ is estimated using a mechanical stylus profilometer (Dektak Veeco). To estimate correctly $h_{d}$, we measured the coating thickness in-between two parallel scratches separated by $\approx 1 \mathrm{~mm}$ performed on the coating in the $x$ direction. For undulating conditions, $h_{d}$ corresponds to the mean thickness, and the typical undulations are of the order of $6 \pm 1.5 \mu \mathrm{m}$, of the order of $15-20 \% h_{d}$.

To estimate the evaporation rate $Q_{e v}$ in our configuration for different $L_{e v}$, we proceed as follows. We glue different glass pieces to form a rectangular tank of width $50 \mathrm{~mm}$, depth $1 \mathrm{~mm}$, and of different lengths $L_{e v}$ ranging from 5 to $10 \mathrm{~mm}$. We then fill the tank to the brim with pure water to mimic the conditions of bladecoating shown in Figure 4(b). The whole setup is then placed on a precision balance, and we record the temporal evolution of its mass over typically 1 hour as well as the external relative humidity and room temperature to further estimate $\beta . Q_{e v}$ is finally estimated from the temporal derivative of such data, and typical uncertainties are of the order of $\simeq 10 \%$.

To estimate the colloid volume fraction $\varphi_{c}$, we acquired top images of the coatings using scanning electron microscopy (SEM, Hitachi TM3030), see Supporting Information for a typical SEM image. We then constructed pair correlation functions $g(r)$ from such images, and 
extracted the surface fraction occupied by the colloids from the first peak of $g(r)$, see ref $^{32}$ for details. Assuming the isotropy of the denselypacked colloidal assembly, we finally estimate $\varphi_{c}=0.7 \pm 0.02$ for different experimental conditions.

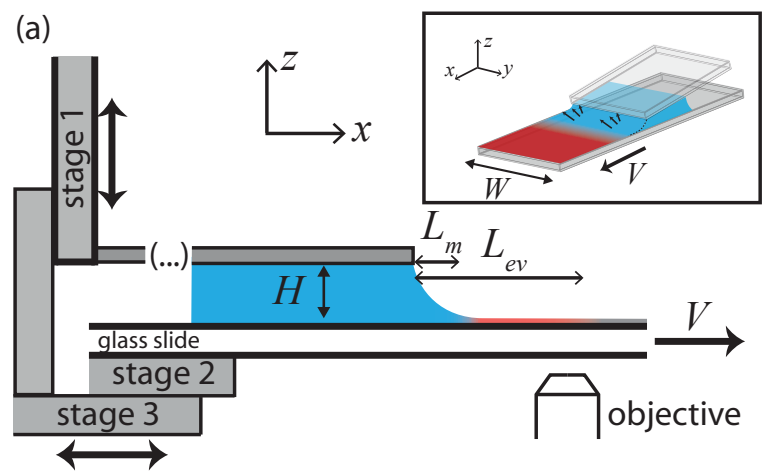

(b)

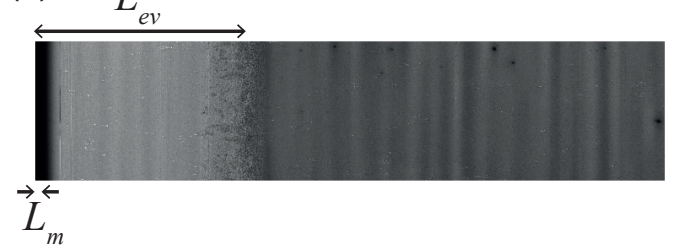

Figure 4: (a) Schematic cross-section of our custom-made blade coating setup mounted on a microscope. The all coating setup is mounted on stage 3 which makes it possible to visualize the film during the coating process at different locations and with the accuracy of optical microscopy. The inset shows schematically a perspective view of the setup. (b) Typical extended image recorded using the setup shown in (a), $L_{e v}=2100 \pm 300 \mu \mathrm{m}$.

In the next section, we include in our analysis measurements of close-packed region length from Jung and Ahn ${ }^{24}$ and Joshi and Gilchrist. ${ }^{21}$ These data were obtained in the following way. Jung and Ahn used a vertical plate immersed in a dilute dispersion of silica particles in ethanol. The plate is immobile, and the relative motion of the contact line is due to solvent evaporation from the bath (the substrate relative velocity is then equal to the mean evaporation velocity). The meniscus length reads $L_{m}=\sqrt{2} \ell_{c}$, where $\ell_{c}$ is the solvent capillary length.

Joshi and Gilchrist ${ }^{21}$ used silica particles in DI water, and the same device as that represented in Figure 1, but with a plate angle larger than $90^{\circ}$. They do not provide any estimation of the evaporation rate. We assumed a meniscus length $L_{m}$ equal to the radius of curvature of the meniscus with zero effective contact angle (see SI of ref ${ }^{33}$ ). $L_{m}$ ranges from $0.3 \mathrm{~mm}$ to $0.9 \mathrm{~mm}$, much lower than $L_{e v}$ in all cases. We disregarded experimental results corresponding to mean deposit thickness lower than particle diameter. Notice that for Jung and Ahn data as well as Gilchrist data, we assumed $\varphi_{c}=0.74$ as these authors did so in their models.

\section{Evaporation length}

Experimental values of the evaporation length $L_{e v}$ are shown in Figure 5(a). The substrate velocity spreads over three and a half decades, and $L_{e v}$ over two decades. Figure 5(b) shows that our data and that of Jung and Ahn collapse on the same master curve, which coincides with the theoretical prediction. Joshi and Gilchrist data are significantly above the master curve, but still obey the scaling law predicted by theory. This discrepancy may be explained by a shift in the estimation of $\mathcal{M}$ due to (i) the uncertainty on $\varphi_{c}$ (Joshi and Gilchrist data collapse on the master curve if we adopt $\varphi_{c} \simeq 0.61$ instead of 0.74 ), (ii) the law used to estimate $\kappa$, the prefactor of the pore-emptying criterion. We used eq (21) as suggested in ref ${ }^{31}$ for a randompacking of monodisperse spheres, but $\kappa$ values ranging from 2 to $\simeq 13$ have been used by different groups, depending on the exact packing and particles wetting properties, see e.g. ref. ${ }^{34}$

Considering that main simplifications in our model lies in the description of vapor mass transfer in the gas phase, these results confirm that the intensity of the overall evaporation rate as well as the shape of the evaporation velocity $v_{e v}(x)$ have only a minor effect on the evaporation length $L_{e v}$. An important result is that eq (26), although based on a simple 1D model of the gas phase, provides a rather fair estimation of $L_{e v}$, provided $\varphi_{c}$ is known.

\section{Deposit thickness}

Experimental data of $h_{d}$ are reported in Figure 6 for our experiments (Jung and Ahn did 
Table 3: Experimental data sets (symbols in the last column are used in Figures 5 and 6).

\begin{tabular}{|c|c|c|c|c|c|}
\hline Source & Set \# & $2 a / \mathrm{nm}$ & $V / \mu \mathrm{m} . \mathrm{s}^{-1}$ & $\varphi_{0}$ & Symbol \\
\hline \multirow{7}{*}{ This work } & 1 & 310 & {$[1,30]$} & 0.048 & 4 \\
\hline & 2 & 310 & {$[0.5,20]$} & 0.0095 & $\triangle$ \\
\hline & 3 & 310 & {$[2,100]$} & 0.096 & $D$ \\
\hline & 4 & 170 & {$[2,100]$} & 0.077 & $\square$ \\
\hline & 5 & 170 & {$[1,50]$} & 0.038 & $\bigcirc$ \\
\hline & 6 & 800 & {$[5,50]$} & 0.096 & $\diamond$ \\
\hline & 7 & 800 & {$[1,20]$} & 0.048 & $\nabla$ \\
\hline \multirow{2}{*}{ Jung and $\mathrm{Ahn}^{24}$} & 8 & $200 \pm 14$ & 0.097 & {$[0.001,0.01]$} & $x$ \\
\hline & 9 & $200 \pm 14$ & {$[0.036,0.097]$} & 0.0025 & + \\
\hline Joshi and Gilchrist ${ }^{21}$ & 10 & $1010 \pm 20$ & {$[27,74]$} & 0.2 & $\bullet$ \\
\hline
\end{tabular}

not perform thickness measurements and data in real units were not available in Joshi and Gilchrist work).

Each symbol corresponds to an experimental data set performed with the same particle diameter and the same reservoir particle volume fraction. From Figure 6(a) it appears that, for each data set, $h_{d}$ is roughly proportional to the inverse of $V$, following scaling (1). In addition, multiplying $h_{d}$ by $\varphi_{0} /\left[\varphi_{c}\left(1-\varphi_{0}\right)\right]$ significantly gathers the data [Figure 6(b)].

The asymptotic models corresponding to scaling (1) predict a negligible (logarithmic) dependence of the deposit thickness on the particle diameter, or no dependence at all. To go further in the comparison between model and data, we define the quantity $\bar{C}$ as the mean value of the expression $h_{d} V \varphi_{c}\left(1-\varphi_{0}\right) / \varphi_{0}$ for each experimental data set, and we plot it in the inset in Figure 6(b) as a function of the particle diameter $2 a$. Despite the dispersion of the data, a weak dependence of $\bar{C}$ with the particle diameter can be observed. This weak dependence is not captured by the model, likely because mass transfer in the gas phase has been oversimplified.

Nevertheless, $h_{d}$ is closer to scaling (1) than scaling (4). In addition, large variations of $L_{e v}$ are observed in Figure 5 for the same sets of control parameters. These large variations of $L_{e v}$ for values of $\mathcal{M} \gg 1$, together with scaling (1) for $h_{d}$ implies that $Q_{e v}$ must depend only weakly on $L_{e v}$ [cf. eq (12)]. To confirm this point, a few measurements of $Q_{e v}$ have been performed (see description above in the Experiment section), in order to investigate its dependence with $L_{e v}$. While $L_{e v}$ has been varied on two orders of magnitude, $Q_{e v}$ increases only by a factor of about 4 (see Figure 7 and SI for detailed comments). This confirms that our experiments are done in a regime such that the evaporation rate $Q_{e v}$ weakly depends on the evaporation length $L_{e v}$. Our experimental results are thus consistent with our theoretical findings.

\section{Conclusion}

In flow coating processes, the use of colloidal dispersions in the evaporative regime deserves special attention, because the evaporation length can be significantly increased by the water flow in the wet porous deposit. In the present work, the effect of the vapor mass transfer on the evaporation length and deposit thickness has been discussed in-depth. We proposed a 2D model simple enough to derive analytical solutions, yet realistic to predict scaling laws observed in experiments.

We conclude from the theoretical analysis that the evaporation length $L_{e v}$ does not depend on the overall evaporation rate, and weakly depends on the shape of the local evap- 

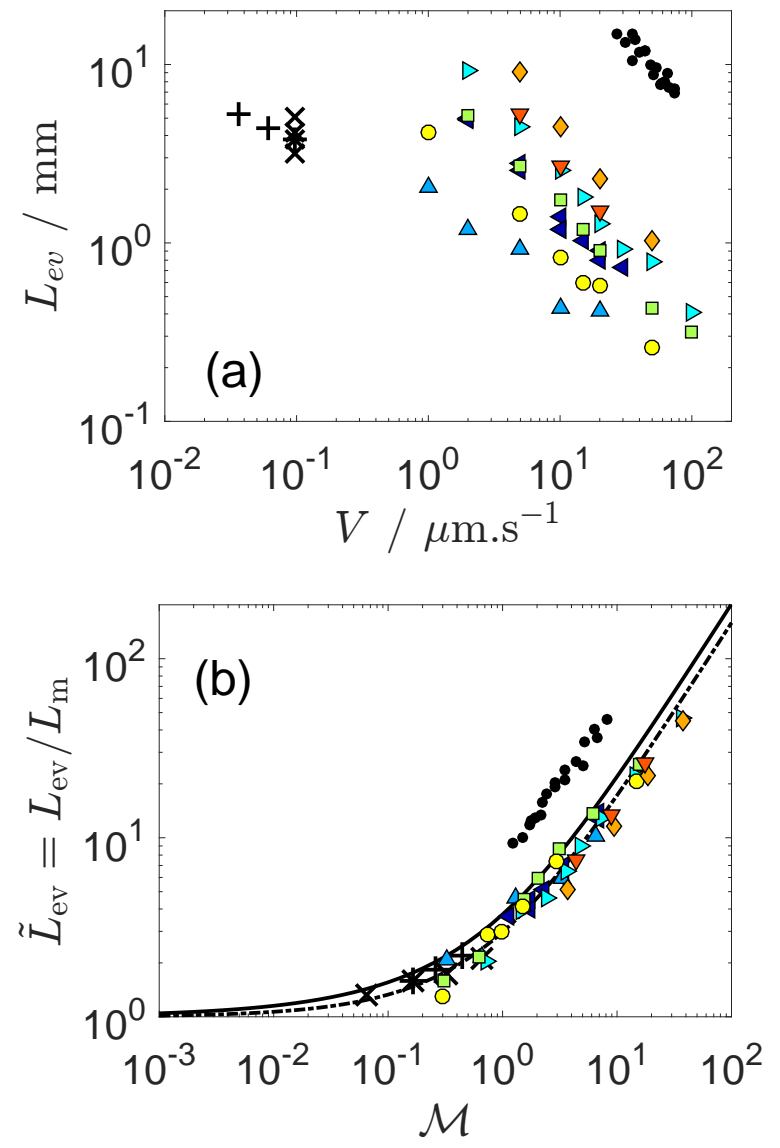

Figure 5: Evaporation length (see Table 3 for symbols). (a) experimental data. (b) master curve $\left(\mathcal{M}\right.$ has been estimated with $\varphi_{c}=0.7$ for our data, and $\varphi_{c}=0.74$ for data from $\left.\operatorname{refs}^{21,24}\right)$; the solid and dashed lines correspond to asymptotic cases $\Lambda / L_{e v} \ll 1[\mathrm{eq}(26)]$ and $\Lambda / L_{e v} \gg 1$ [eq (28)], respectively.
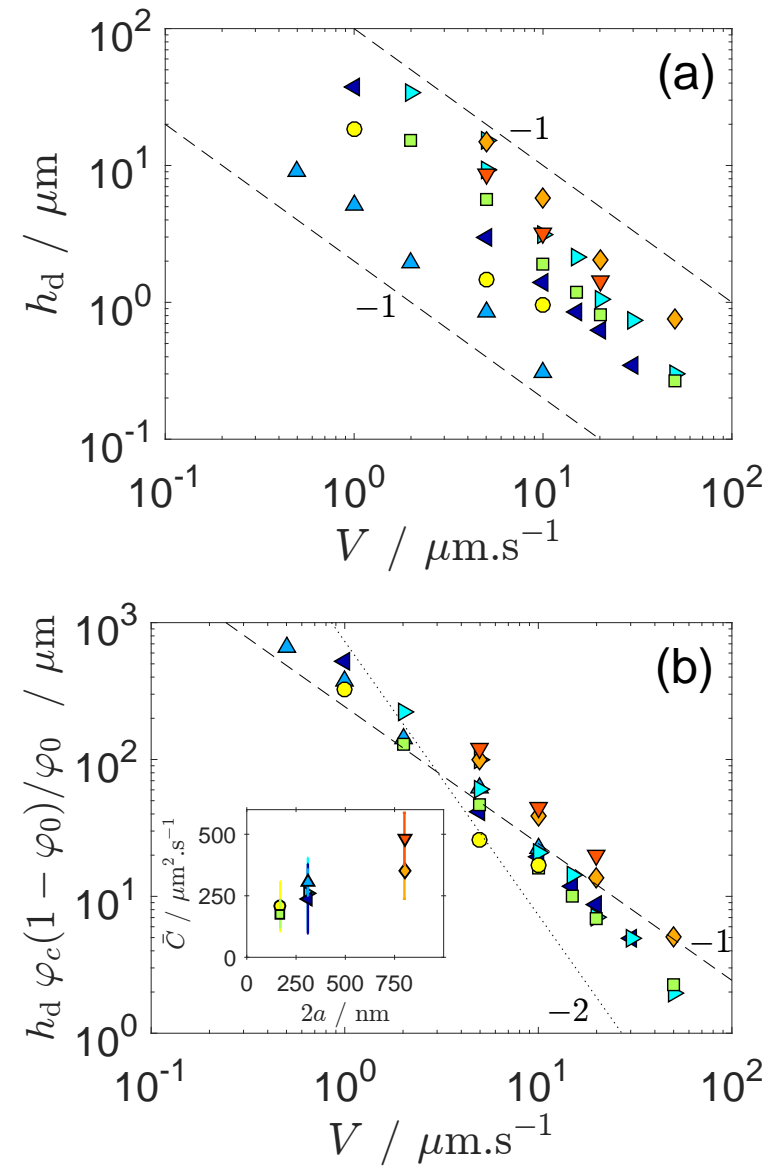

Figure 6: Experimental deposit height. (a) $h_{d}$ and (b) $h_{d} \varphi_{c}\left(1-\varphi_{0}\right) / \varphi_{0}$ as a function of substrate velocity $V$. Dashed and dotted lines are power laws with -1 and -2 exponents, respectively. See Table 3 for symbols. Inset : parameter $\bar{C}$ defined as the mean value of the expression $h_{d} V \varphi_{c}\left(1-\varphi_{0}\right) / \varphi_{0}$ for each experimental data set, as a function of particle diameter $2 a$ (errorbars represent the standard deviations). 


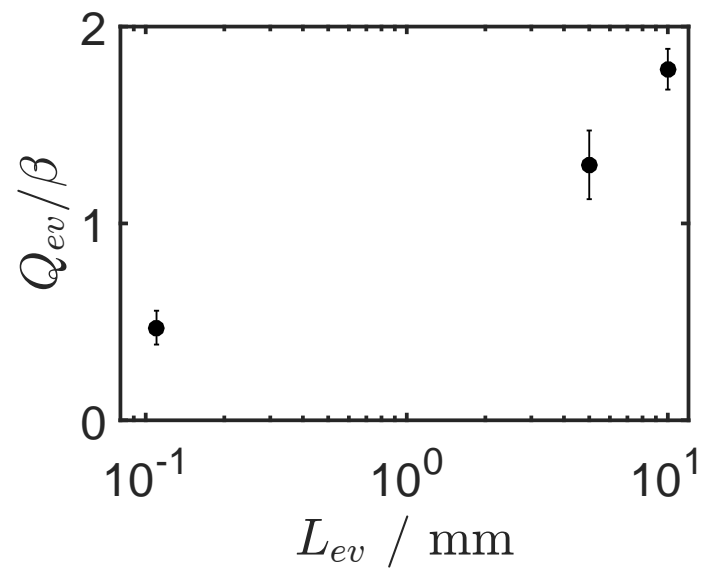

Figure 7: Normalized experimental evaporation rate $Q_{e v} / \beta$ as a function of the evaporation length $L_{e v}$.

oration velocity. For this reason, a model based on $1 \mathrm{D}$ vapor mass transfer still provides fair estimates of $L_{e v}$, when compared to experimental data obtained in a 3D configuration.

On the contrary, an accurate model of vapor mass transfer is required for the prediction of the deposit thickness $h_{d}$, in order to describe precisely how the overall evaporation rate $Q_{e v}$ depends on the evaporation length $L_{e v}$ (this is specific to colloidal dispersions, as $L_{e v}$ slightly varies for polymers or small solutes solutions $\left.{ }^{35}\right)$. The scaling law relating $h_{d}$ to process control parameters strongly depends on the driving mechanism acting in a given experimental setup. We believe that scalings (4) and (1) are two limiting cases, the latter corresponding to weak variations of $Q_{e v}$ with $L_{e v}$, as expected for diffusion in semi-infinite quiescent air (essentially a 3D process), the former resulting from proportionality between $Q_{e v}$ and $L_{e v}$, as would be expected for instance for turbulent free convection. Intermediate scalings should be possible with other vapor mass transfer mechanisms.

Our theoretical work also demonstrates that the comparison between measurements of $L_{e v}$ and the predictions given in Table 1 , is a possible way to estimate $\mathcal{M}$, the dimensionless parameter defined by eq (24). Such estimates are quite robust as our model shows that $L_{e v}$ does not depend on the global evaporation rate, and is nearly insensitive to the distribution of the local evaporation flux. Our measurements are thus an indirect way to estimate precisely the product of the pore pressure at poreopening with the value of the permeability of the densely-packed wet film, see eq (24). This quantity is key to predict the receding of water during horizontal drying of films or sessile drops, ${ }^{20,23,34}$ but also for all unidirectional geometries such as the 1D drying of gels or of dispersions confined within capillaries. ${ }^{36-38}$

Acknowledgement The authors thank ANR EVAPEC (13-BS09-0010-01) for funding. FD and BG thank Jean-Pierre Hulin, from FAST laboratory, for useful discussions. We thank Jacques Leng for his analysis of the SEM images to estimate $\varphi_{c}$.

Supporting Information Available: Diffusion evaporation flux for an infinite stripe domain - SEM top view of coating - Driving mechanism of the evaporation flux in the experimental device. This material is available free of charge via the Internet at http: //pubs.acs.org/.

\section{References}

(1) Sondergaard, R.; Hösel, M.; Angmo, D.; Larsen-Olsen, T.; Krebs, F. C. Roll-to-roll fabrication of polymer solar cells. Mater. Today 2012, 15, 36-49.

(2) Gu, X.; Yan, H.; Kurosawa, T.; Schroeder, B. C.; Gu, K. L.; Zhou, Y.; to, J. W. F.; Oosterhout, S. D.; Savikhin, V.; Molina-Lopez, F.; Tassone, C. J.; Mannsfeld, S. C. B.; Wang, C.; Toney, M. F.; Bao, Z. Comparison of the Morphology Development of PolymerFullerene and Polymer-Polymer Solar Cells during Solution-Shearing Blade Coating. Adv. Energy Mater. 2016, 6, 1601225.

(3) Prevo, B. G.; Kuncicky, D. M.; Velev, O. D. Engineered deposition of coatings from nano- and micro-particles: A brief review of convective assembly at 
high volume fraction. Colloids Surf., A 2007, 311, 2-10.

(4) Le Berre, M.; Chen, Y.; Baigl, D. From convective assembly to Landau-Levich deposition of multilayered phospholipid films of controlled thickness. Langmuir 2009, 25, 2554-2557.

(5) Grosso, D. How to exploit the full potential of the dip-coating process to better control film formation. J. Mater. Chem. 2011, 21, 17033-17038.

(6) Berteloot, G.; Pham, C.-T.; Daerr, A.; Lequeux, F.; Limat, L. Evaporationinduced flow near a contact line: Consequences on coating and contact angle. Europhys. Lett. 2008, 83, 14003.

(7) Weinstein, S.; Ruschak, K. Coating Flows. Annu. Rev. Fluid Mech. 2004, 36, 29-53.

(8) Davis, R.; Jayaraman, S.; Chaikin, P.; Register, R. Creating Controlled Thickness Gradients in Polymer Thin Films via Flowcoating. Langmuir 2014, 30, 56375644 .

(9) Dimitrov, A. S.; Nagayama, K. Continuous Convective Assembling of Fine Particles into Two-Dimensional Arrays on Solid Surfaces. Langmuir 1996, 12, 1303-1311.

(10) Berteloot, G.; Daerr, A.; Lequeux, F.; Limat, L. Dip coating with colloids and evaporation. Chem. Eng. Proc. 2013, 68, 69-73.

(11) Prevo, B. G.; Velev, O. D. Controlled, Rapid Deposition of Structured Coatings from Micro- and Nanoparticle Suspensions. Langmuir 2004, 20, 2099.

(12) Kuncicky, D.; Naik, R.; Velev, O. Rapid Deposition and Long-Range Alignment of Nanocoatings and Arrays of Electrically Conductive Wires from Tobacco Mosaic Virus. Small 2006, 2, 1462-1466.

(13) Brewer, D. D.; Shibuta, T.; Francis, L.; Kumar, S.; Tsapatsis, M. Coating Process
Regimes in Particulate Film Production by Forced-Convection-Assisted Drag-Out. Langmuir 2011, 27, 11660.

(14) Jing, G.; Bodiguel, H.; Doumenc, F.; Sultan, E.; Guerrier, B. Drying of Colloidal Suspensions and Polymer Solutions near the Contact Line: Deposit Thickness at Low Capillary Number. Langmuir 2010, 26, 2288-2293.

(15) Maloy, S. F.; Martin, G. L.; Atanassov, P.; Cooney, M. J. Controlled Deposition of Structured Polymer Films: Chemical and Rheological Factors in Chitosan Film Formation. Langmuir 2012, 28, 2589-2595.

(16) Diao, Y.; Tee, B. C.-K.; Giri, G.; $\mathrm{Xu}$, J.; Kim, D. H.; Becerril, H. A.; Stoltenberg, R. M.; Lee, T. H.; Xue, G.; Mannsfeld, S. C. B.; Bao, Z. Solution coating of large-area organic semiconductor thin films with aligned single-crystalline domains. Nat. Mater. 2013, 12, 665-671.

(17) Bodiguel, H.; Doumenc, F.; Guerrier, B. Stick- slip patterning at low capillary numbers for an evaporating colloidal suspension. Langmuir 2010, 26, 1075810763.

(18) Mino, Y.; Watanabe, S.; Miyahara, M. T. In Situ Observation of Meniscus Shape Deformation with Colloidal Stripe Pattern Formation in Convective Self-Assembly. Langmuir 2015, 31, 4121-4128.

(19) Boettcher, J. M.; Joy, M.; Joshi, K.; Muangnapoh, T.; Gilchrist, J. F. Spacing of seeded and spontaneous streaks during convective deposition. Langmuir 2015, 31, 10935.

(20) Routh, A. F. Drying of thin colloidal films. Rep. Prog. Phys. 2013, 76, 046603.

(21) Joshi, K.; Gilchrist, J. Estimation of drying length during particle assembly by convective deposition. J. Colloid Interface Sci. 2017, 496, 222 - 227. 
(22) Routh, A. F.; Russel, W. B. Horizontal Drying Evaporation Fronts During Solvent from Latex Films. AIChE J. 1998, 44, 2088-2098.

(23) Goehring, L.; Clegg, W. J.; Routh, A. F. Solidification and ordering during directional drying of a colloidal dispersion. Langmuir 2010, 26, 9269-9275.

(24) Jung, Y. D.; Ahn, K. H. Prediction of Coating Thickness in the Convective Assembly Process. Langmuir 2013, 29, 15762-15769.

(25) Doumenc, F.; Salmon, J. B.; Guerrier, B. Modeling flow coating of colloidal dispersions in the evaporative regime: prediction of deposit thickness. Langmuir 2016, 32, 13657-13668.

(26) Rhosenow, W.; Hartnett, J.; Cho, Y. Handbook of Heat Transfer, 3rd ed.; McGraw-Hill: New York, USA, 1998.

(27) Deegan, R. D.; Bakajin, O.; Dupont, T. F.; Huber, G.; Nagel, S. R.; Witten, T. Contact line deposits in an evaporating drop. Phys. Rev. E 2000, 62, 756 .

(28) Janeček, V.; Doumenc, F.; Guerrier, B.; Nikolayev, V. Can hydrodynamic contact line paradox be solved by evaporationcondensation? J. Colloid Interface Sci. 2015, 460, $329-338$.

(29) Guyon, E.; Hulin, J. P.; Petit, L.; Mitescu, C. D. Physical Hydrodynamics; Oxford University Press, 2015.

(30) Zick, A. A.; Homsy, G. M. Stokes flow through periodic arrays of spheres. $J$. Fluid Mech. 1982, 115, 13-26.

(31) Brown, L. A.; Zukoski, C. F.; White, L. R. Consolidation During Drying of Aggregated Suspensions. AIChE J. 2002, 48, 492-502.

(32) Angly, J.; Iazzolino, A.; Salmon, J. B.; Leng, J.; Chandran, S.; Ponsinet, V.;
Desert, A.; Beulze, A. L.; Mornet, S.; Treguer-Delapierre, M.; CorreaDuarte, M. Microfluidic-induced growth and shape-up of three-dimensional extended arrays of densely packed nanoparticles. ACS Nano 2013, 7, 6465.

(33) Kumnorkaew, P.; Ee, Y.-K.; Tansu, N.; Gilchrist, J. F. Investigation of the Deposition of Microsphere Monolayers for Fabrication of Microlens Arrays. Langmuir 2008, 24, 12150-12157.

(34) Keddie, J. L.; Routh, A. F. Fundamentals of latex film formation. Processes and Properties; Springer, 2010.

(35) Doumenc, F.; Guerrier, B. Drying of a Solution in a Meniscus: A Model Coupling the Liquid and the Gas Phases. Langmuir 2010, 26, 13959-13967.

(36) Brinker, C. J.; Scherer, G. W. Sol-Gel Science: The Physics and Chemistry of SolGel Processing; Academic Press, 1990.

(37) Dufresne, E. R.; Corwin, E. I.; Greenblatt, N. A.; Ashmore, J.; Wang, D. Y.; Dinsmore, A. D.; Cheng, J. X.; Xie, X.; Hutchinson, J. W.; Weitz, D. A. Flow and fracture in drying nanoparticle suspensions. Phys. Rev. Lett. 2003, 91, 224501.

(38) Lidon, P.; Salmon, J. B. Dynamics of unidirectional drying of colloidal dispersions. Soft Matter 2014, 10, 4151. 


\section{Graphical TOC Entry}

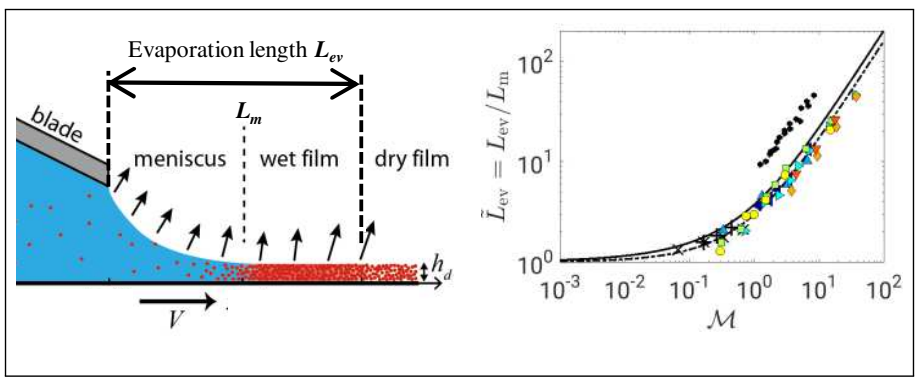

